International Journal of Pure and Applied Mathematics

Volume 108 No. 4 2016, 985-997

ISSN: 1311-8080 (printed version); ISSN: 1314-3395 (on-line version)

url: http://www.ijpam.eu

doi: 10.12732/ijpam.v108i4.22

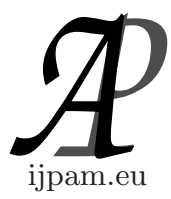

\title{
ON THE OFFSETS OF RULED SURFACES IN EUCLIDEAN SPACE
}

\author{
Dae Won Yoon \\ Department of Mathematics Education and RINS \\ Gyeongsang National University \\ Jinju 52828, REPUBLIC OF KOREA
}

\begin{abstract}
In the present paper, we study evolute offsets of a non-developable ruled surface in Euclidean 3-space $\mathbb{E}^{3}$ and classify the evolute offset with constant Gaussian curvature and constant mean curvature. In last section, we investigate linear Weingarten evolute offsets in $\mathbb{E}^{3}$. A linear Weingarten surface is the surface having a linear equation between the Gaussian curvature and the mean curvature of a surface.
\end{abstract}

AMS Subject Classification: 53C30, 53B25

Key Words: linear Weingarten surface, offset, constant curvature surface, ruled surface

\section{Introduction}

A surface $M$ in Euclidean 3 -space $\mathbb{E}^{3}$ is called a Weingarten surface if there exists a non-trivial differentiable function relating the Gaussian curvature $K$ and the mean curvature $H$ of a surface $M$. Also, if a surface $M$ satisfies a linear equation with respect to $K$ and $H$, that is, $a K+b H=c$ for some real numbers $a, b, c$ not all zero, then it is said to be a linear Weingarten surface. Some typical examples of Weingarten surfaces and linear Weingarten surfaces are constant mean curvature surfaces and constant Gaussian curvature surfaces.

The study of the Weingarten surface is initiated by Weingarten in 1861 [18], and taken up by Beltrami [3], Darboux [5], Dini [6] and Lie [12], and so on. Several geometers have studied Weingarten surfaces and linear Weingarten

Received: February 16, 2016

Published: August 16, 2016 (c) 2016 Academic Publications, Ltd.

url: www.acadpubl.eu 
surfaces in the ambient spaces, and many interesting results have been obtained. The ruled Weingarten surfaces in $\mathbb{E}^{3}$ were classified by Kühnel [11]. For the another space forms $\mathbb{S}^{3}$ and $\mathbb{H}^{3}$ Valério was studied in [17], but a complete classification is not known yet. Following this line of reasoning, the classification of ruled Weingarten hypersurfaces in $\mathbb{E}^{n+1}, \mathbb{S}^{n+1}$ and $\mathbb{H}^{n+1}, n \geq 3$ is obtained in [1], [4] and [15], respectively. On the other hand, the linear Weingarten surfaces are examined in [7], [14], [16] and [19], etc. In [14], Lopez investigated rotational linear Weingarten surfaces of hyperbolic type in Euclidean 3-space $\mathbb{E}^{3}$ and he proved that the profile curves of these surfaces are periodic and have selfintersections. Also, Barros, Silva and Sousa [2] gave a complete description of all rotational linear Weingarten surfaces in Euclidean 3 -sphere $\mathbb{S}^{3}$. The present author, Tuncer and Karacan [16], [19] completely classified tubular surfaces and quadric surfaces in $\mathbb{E}^{3}$.

In this paper, we study offsets of ruled surfaces in Euclidean 3-space $\mathbb{E}^{3}$. Also we study an evolute offset with constant Gaussian curvature and constant mean curvature and give examples. As the results, we classify a linear Weingarten evolute offset of ruled surfaces.

\section{Preliminaries}

Let $\gamma: I \longrightarrow \mathbb{E}^{3}$ be a regular space curve in Euclidean 3 -space $\mathbb{E}^{3}$ parameterized by its arc-length $s$. For the Frenet frame field $\{\mathbf{t}, \mathbf{n}, \mathbf{b}\}$ along $\gamma(s)$, the Frenet formulas of $\gamma(s)$ are given by

$$
\begin{aligned}
\dot{\gamma}(s) & =\mathbf{t}(s) \\
\dot{\mathbf{t}}(s) & =\kappa(s) \mathbf{n}(s) \\
\dot{\mathbf{n}}(s) & =-\kappa(s) \mathbf{t}(s)+\tau(s) \mathbf{b}(s), \\
\dot{\mathbf{b}}(s) & =-\tau(s) \mathbf{n}(s)
\end{aligned}
$$

The functions $\kappa(s)$ and $\tau(s)$ are the curvature function and torsion function of $\gamma(s)$. Here "dot" denotes the derivative with respect to $s$.

If $\gamma(s)$ is a spherical curve in $\mathbb{E}^{3}$ with $\langle\gamma(s), \gamma(s)\rangle=1$. Let $\mathbf{t}(s)=\dot{\gamma}(s)$ and $\mathbf{g}(s)=\gamma(s) \times \dot{\gamma}(s)$. Then $\{\gamma(s), \mathbf{t}(s), \mathbf{g}(s)\}$ is an orthonormal frame along the curve $\gamma(s)$ in $\mathbb{E}^{3}$ and it is called the spherical Frenet frame of the spherical curve $\gamma(s)$ in $\mathbb{E}^{3}$. On the other hand, for the spherical Frenet frame we can 
obtain the following formulas:

$$
\begin{aligned}
\dot{\gamma}(s) & =\mathbf{t}(s), \\
\dot{\mathbf{t}}(s) & =-\gamma(s)-\kappa_{g} \mathbf{g}(s), \\
\dot{\mathbf{g}}(s) & =\kappa_{g} \mathbf{t}(s) .
\end{aligned}
$$

The function $\kappa_{g}$ is called the geodesic curvature of the spherical curve $\gamma(s)$ in $\mathbb{E}^{3}$. We have the following conclusions (see [9], [10]).

Proposition 1. The geodesic curvature $\kappa_{g}(s)$, the curvature $\kappa(s)$ and the torsion $\tau(s)$ of a spherical curve $\gamma(s)$ in $\mathbb{E}^{3}$ satisfy

$$
\begin{aligned}
\kappa(s) & =\sqrt{1+\kappa_{g}^{2}(s)}, \\
\tau(s) & =\frac{ \pm \dot{\kappa}_{g}(s)}{\kappa_{g}^{2}(s)+1} .
\end{aligned}
$$

Proposition 2. If the geodesic curvature $\kappa_{g}(s)$ of a spherical curve $\gamma(s)$ in $\mathbb{E}^{3}$ is constant, $\gamma(s)$ is a circle.

Remark 1. If the geodesic curvature $\kappa_{g}(s)$ of a spherical curve $\gamma(s)$ is zero identically, $\gamma(s)$ is a great circle on the unit sphere.

\section{Offsets with Constant Gaussian Curvature and Mean Curvature}

Let $M$ be a non-developable ruled surface in $\mathbb{E}^{3}$. Then the parametrization for $M$ is given by

$$
\varphi(u, v)=\mathbf{c}(u)+v \mathbf{e}(u),
$$

where $\langle\mathbf{e}(u), \mathbf{e}(u)\rangle=1,\left\langle\mathbf{e}^{\prime}(u), \mathbf{e}^{\prime}(u)\right\rangle=1$ and the directrix $\mathbf{c}(u)$ is the striction curve of the surface $\varphi(u, v)$. Here the prime denotes derivative with respect to $u$. Such parametrization of $\varphi(u, v)$ is called standard equation of the nondevelopable ruled surfaces in Euclidean 3 -space $\mathbb{E}^{3}$ (see [13]). In the case, the parameter $u$ is the arc-length parameter of $\mathbf{e}(u)$ as a spherical curve in $\mathbb{E}^{3}$. Also, $\mathbf{e}(u)$ can be regarded as a vector and it called the spherical indicatrix vector of $\varphi(u, v)$. Since $\mathbf{c}(u)$ is the striction curve of $\varphi(u, v),\left\langle\mathbf{c}^{\prime}(u), \mathbf{e}^{\prime}(u)\right\rangle=0$.

From now on, we shall often not write the parameter $u$ explicitly in our formulas. We put $\mathbf{t}=\mathbf{e}^{\prime}$ and $\mathbf{g}=\mathbf{e} \times \mathbf{e}^{\prime}$. Then the set $\{\mathbf{e}, \mathbf{t}, \mathbf{g}\}$ is the spherical Frenet frame of $\mathbf{e}$ and the vectors $\mathbf{t}$ and $\mathbf{g}$ are said to be the central normal and 
the asymptotic normal of $\varphi(u, v)$, respectively. For the spherical Frenet frame $\{\mathbf{e}, \mathbf{t}, \mathbf{g}\}$, the following equations hold:

$$
\begin{aligned}
\mathbf{e}^{\prime} & =\mathbf{t}, \\
\mathbf{t}^{\prime} & =-\mathbf{e}-J \mathbf{g}, \\
\mathbf{g}^{\prime} & =J \mathbf{t},
\end{aligned}
$$

where $J=\left\langle\mathbf{e}^{\prime \prime}, \mathbf{e}^{\prime} \times \mathbf{e}\right\rangle$ denotes the geodesic curvature $\kappa_{g}$ of a spherical indicatrix curve e.

On the other hand, the derivative of the striction curve $\mathbf{c}$ is given by

$$
\mathbf{c}^{\prime}=F \mathbf{e}+Q \mathbf{g},
$$

where $F=\left\langle\mathbf{c}^{\prime}, \mathbf{e}\right\rangle$ and $Q=\left\langle\mathbf{c}^{\prime}, \mathbf{e} \times \mathbf{e}^{\prime}\right\rangle$. The function $Q$ is called the parameter of distribution of $\varphi(u, v)$. The functions $J, F$ and $Q$ of $\varphi(u, v)$ are called structure functions of a non-developable ruled surface $\varphi(u, v)$ in Euclidean 3 -space $\mathbb{E}^{3}$.

Proposition 3. ([13]) Let $\varphi(u, v)$ be a non-developable ruled surface with the standard equation in Euclidean 3-space $\mathbb{E}^{3}$. Then $\varphi(u, v)$ is uniquely determined by its structure functions $J, F$ and $Q$, up to orthonormal transformations in $\mathbb{E}^{3}$.

On the other hand, the parameter $u$ is the arc-length parameter of the curve e, but usually $u$ is not the arc-length parameter of the curve c. By (3.3), we have

Proposition 4. Let $\varphi(u, v)$ be a non-developable ruled surface with the standard equation in $\mathbb{E}^{3}$. If the parameter $u$ is also the arc-length parameter of the striction curve $\mathbf{c}$ of $\varphi(u, v)$, the structure functions $F$ and $Q$ of $\varphi(u, v)$ satisfy $F^{2}+Q^{2}=1$.

Now, we compute the Gaussian curvature and the mean curvature of a non-developable ruled surface $\varphi(u, v)$ in $\mathbb{E}^{3}$. From (3.1), (3.2) and (3.3) the coefficients of the first fundamental form of $\varphi(u, v)$ are given by

$$
E=F^{2}+Q^{2}+v^{2}, F=\left\langle\mathbf{c}^{\prime}, \mathbf{e}\right\rangle, G=1 .
$$

And, the unit normal vector $\mathbf{u}$ of $\varphi(u, v)$ is written as

$$
\mathbf{u}=\frac{1}{D}\left(\mathbf{c}^{\prime} \times \mathbf{e}+v \mathbf{e}^{\prime} \times \mathbf{e}\right)=\frac{1}{D}(Q \mathbf{t}-v \mathbf{g}),
$$

where $D=\sqrt{E G-F^{2}}=\sqrt{Q^{2}+v^{2}}$. This leads to the coefficients $L, M$ and $N$ of the second fundamental form as

$$
L=\frac{1}{D}\left(Q(F+Q J)-Q^{\prime} v+J v^{2}\right), M=\frac{Q}{D}, N=0 .
$$


Thus, using the data described above, the Gaussian curvature $K$ and the mean curvature $H$ of $\varphi(u, v)$ are given respectively by

$$
\begin{aligned}
& K=-\frac{Q^{2}}{D^{4}} \\
& H=\frac{1}{2 D^{3}}\left(J v^{2}-Q^{\prime} v+Q(Q J-F)\right) .
\end{aligned}
$$

Since $\varphi(u, v)$ is a non-developable ruled surface, the function $Q$ is non-zero everywhere.

Definition 2. Let $\varphi(u, v)$ and $\varphi^{*}(u, v)$ be two ruled surfaces in $\mathbb{E}^{3}$. The surface $\varphi(u, v)$ is said to be an involute offset of $\varphi^{*}(u, v)$ if there exists a oneto-one correspondence between their rulings such that the central normal of $\varphi(u, v)$ and the spherical indicatrix vector of $\varphi^{*}(u, v)$ are linearly dependent at the striction points of their corresponding rulings. In the case, $\varphi^{*}(u, v)$ is said to be an evolute offset of $\varphi(u, v)$.

Let $\varphi^{*}(u, v)$ be an evolute offset of a non-developable ruled surface $\varphi(u, v)$ with the standard equation in Euclidean 3 -space $\mathbb{E}^{3}$. Then the surface $\varphi^{*}(u, v)$ can be written as [8]

$$
\varphi^{*}(u, v)=\mathbf{c}^{*}(u)+v \mathbf{e}^{*}(u)=\mathbf{c}(u)+R(u) \mathbf{t}(u)+v \mathbf{t}(u)
$$

where $R$ is the distance between the corresponding striction points of $\varphi(u, v)$ and $\varphi^{*}(u, v)$. By using (3.2) and (3.3) the coefficients of the first fundamental form of $\varphi^{*}(u, v)$ are

$$
\begin{aligned}
& E^{*}=F^{2}+Q^{2}-2(F+J Q)(R+v)+\left(1+J^{2}\right)(R+v)^{2}+R^{\prime 2}, \\
& F^{*}=R^{\prime} \\
& G^{*}=1 .
\end{aligned}
$$

Also, the unit normal vector $\mathbf{u}^{*}$ of $\varphi^{*}(u, v)$ is given by

$$
\mathbf{u}^{*}=\frac{1}{D^{*}}[(-Q+J R+J v) \mathbf{e}+(F-R-v) \mathbf{g}]
$$

where $D^{*}=\sqrt{E^{*} G^{*}-F^{* 2}}=\sqrt{(-Q+J R+J v)^{2}+(F-R-v)^{2}}$. From this, 
we get the coefficients of the second fundamental form as follows:

$$
\begin{aligned}
L^{*}= & \frac{1}{D^{*}}\left[\left(F^{\prime}-2 R^{\prime}\right)(-Q+J R+J v)\right. \\
& \left.\quad+(F-R-v)\left(Q^{\prime}-2 R^{\prime} J-(R+v) J^{\prime}\right)\right], \\
M^{*}= & \frac{1}{D^{*}}(Q-F J), \\
N^{*}= & 0 .
\end{aligned}
$$

By a direct computation, we can show that the Gaussian curvature $K^{*}$ and the mean curvature $H^{*}$ of $\varphi^{*}(u, v)$ are given by

$$
K^{*}=-\frac{1}{D^{* 4}}(Q-F J)^{2}
$$

and

$$
H^{*}=\frac{1}{2 D^{* 3}} H_{1}^{*},
$$

where $H_{1}^{*}=J^{\prime} v^{2}+\left(F^{\prime} J-F J^{\prime}+2 R J^{\prime}-Q^{\prime}\right) v+\left(-F^{\prime} Q+F Q^{\prime}+F^{\prime} J R-F J^{\prime} R-\right.$ $\left.R Q^{\prime}+R^{2} J^{\prime}\right)$.

Theorem 3. Let $\varphi^{*}(u, v)$ be an evolute offset of a non-developable ruled surface $\varphi(u, v)$. If $\varphi^{*}(u, v)$ has a constant Gaussian curvature $K_{0}^{*}$, then $\varphi^{*}(u, v)$ has a zero Gaussian curvature. Furthermore, the structure functions $Q, J$ and $F$ of $\varphi(u, v)$ satisfy $Q=J F$.

Proof. From (3.6) and the definition of $D^{*}$, we find a polynomial in $v$ with functions of $u$ as coefficients as follows:

$$
K_{0}^{*} D^{* 4}+(Q-F J)^{2}=0
$$

and all the coefficients must be zero. In this case, the coefficient of $v^{4}$ is $\left(J^{2}+\right.$ $1)^{2} K_{0}^{*}$, from this we can obtain $K_{0}^{*}=0$.

Remark 4. Let $\varphi(u, v)=\mathbf{e}^{\prime}(u)+v \mathbf{e}(u)$ be a non-developable ruled surface with $\mathbf{c}(u)=\mathbf{e}^{\prime}(u),\langle\mathbf{e}(u), \mathbf{e}(u)\rangle=1$ and $\left\langle\mathbf{e}^{\prime}(u), \mathbf{e}^{\prime}(u)\right\rangle=1$. Then, we have $F=$ -1 and $Q=-J$. Since $\varphi(u, v)$ is a non-developable ruled surface, the surface $\varphi(u, v)$ has a non-zero Gaussian curvature, but the evolute offset $\varphi^{*}(u, v)$ of $\varphi(u, v)$ has a zero Gaussian curvature, that is, it is flat.

Example 1. We consider $\mathbf{e}(u)=(x(u), y(u), z(u))$ with $\langle\mathbf{e}(u), \mathbf{e}(u)\rangle=1$ and $\left\langle\mathbf{e}^{\prime}(u), \mathbf{e}^{\prime}(u)\right\rangle=1$. Then the following relations hold:

$$
x^{2}+y^{2}+z^{2}=1
$$




$$
x^{\prime 2}+y^{\prime 2}+z^{\prime 2}=1
$$

We now try to solve the above equations. From (3.8), we may put $x=x(u)$ and $y=y(u)$ by

$$
\begin{aligned}
& x(u)=\sqrt{1-z^{2}} \cos \theta(u), \quad-1<z<1 \\
& y(u)=\sqrt{1-z^{2}} \sin \theta(u),
\end{aligned}
$$

and then determine the function $\theta=\theta(u)$ satisfying (3.9). By using (3.9) and (3.10) we have

$$
\theta^{2}=\frac{1-z^{2}-z^{2}}{\left(1-z^{2}\right)^{2}}
$$

We assume that $1-z^{2}-z^{\prime 2}>0$ (when $1-z^{2}-z^{\prime 2}=0, \theta$ is constant). Then, the function $\theta(u)$ is of the form

$$
\theta(u)= \pm \int_{0}^{u} \frac{\sqrt{1-z(t)^{2}-z^{\prime}(t)^{2}}}{1-z(t)^{2}} d t
$$

and without loss of generality we may assume that the signature is positive. Since $z^{2}+z^{\prime 2}<1$, we take $z(u)=\frac{1}{\sqrt{2}} \cos u$. Then we have

$$
\theta(u)=\tan ^{-1}(\sqrt{2} \tan u)
$$

From this, the spherical curve e $(u)$ can be expressed as

$$
\begin{aligned}
\mathbf{e}(u)= & \left(\frac{\sqrt{4-2 \cos ^{2} u}}{2} \cos \left(\tan ^{-1}(\sqrt{2} \tan u)\right)\right. \\
& \left.\frac{\sqrt{4-2 \cos ^{2} u}}{2} \sin \left(\tan ^{-1}(\sqrt{2} \tan u)\right), \frac{\sqrt{2}}{2} \cos u\right) .
\end{aligned}
$$

Thus, the ruled surface $\varphi(u, v)=\mathbf{e}^{\prime}(u)+v \mathbf{e}(u)$ has a non-zero Gaussian curvature, but from Remark 3.5, the evolute offset $\varphi^{*}(u, v)$ of $\varphi(u, v)$ has a zero Gaussian curvature, that is, it is flat.

Next, we consider an evolute offset $\varphi^{*}(u, v)$ with constant mean curvature $H_{0}^{*}$. Then, from (3.7) we have the following equation:

$$
H_{0}^{* 2} D^{* 6}-H_{1}^{* 2}=0
$$




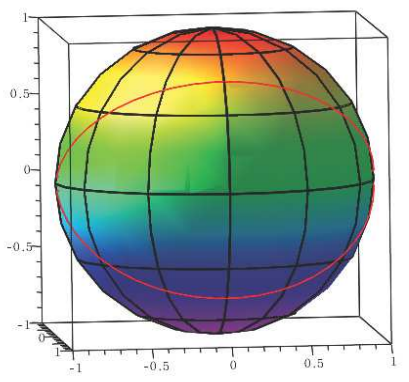

Figure 1: A red line is the spherical curve given by (3.12).

and it is a polynomial in $v$ with functions of $u$ as coefficients. In this case, the coefficient of $v^{6}$ is $\left(J^{2}+1\right)^{3} H_{0}^{* 2}$, which implies $H_{0}^{*}=0$. Thus we show that

$$
\begin{aligned}
F Q^{\prime} & =F^{\prime} Q, \\
Q^{\prime} & =F^{\prime} J, \\
J^{\prime} & =0 .
\end{aligned}
$$

Since $J$ is constant, from (3.2) we can obtain the ODE, $\mathbf{t}^{\prime \prime}+\left(1+J^{2}\right) \mathbf{t}=0$. Without loss of generality, we may assume $\mathbf{t}(0)=(1,0,0)$, the general solution of the ODE becomes

$$
\begin{aligned}
\mathbf{t}(u)= & \left(\cos \left(\sqrt{1+J^{2}} u\right)+B_{1} \sin \left(\sqrt{1+J^{2}} u\right), B_{2} \sin \left(\sqrt{1+J^{2}} u\right),\right. \\
& \left.B_{3} \sin \left(\sqrt{1+J^{2}} u\right)\right)
\end{aligned}
$$

for some constants $B_{1}, B_{2}$ and $B_{3}$. Since $\langle\mathbf{t}, \mathbf{t}\rangle=1$, we get $B_{1}=0$ and $B_{2}^{2}+B_{3}^{2}=$ 1 , it follows that the spherical indicatrix vector of $\varphi(u, v)$ is given by

$$
\mathbf{e}(u)=\left(\frac{1}{k} \sin (k u), \frac{-B_{2}}{k} \cos (k u)+D_{2}, \frac{-B_{3}}{k} \cos (k u)+D_{3}\right)
$$

for some constants $D_{2}, D_{3}$ satisfying $B_{2} D_{2}+B_{3} D_{3}=0$ and $D_{2}^{2}+D_{3}^{2}=\frac{k^{2}-1}{k^{2}}$, where $k=\sqrt{1+J^{2}}$. We now change the coordinates by $\bar{x}, \bar{y}, \bar{z}$ such that $\bar{x}=x$, $\bar{y}=-B_{2} y-B_{3} z, \bar{z}=B_{3} y-B_{2} z$, that is,

$$
\left(\begin{array}{l}
\bar{x} \\
\bar{y} \\
\bar{z}
\end{array}\right)=\left(\begin{array}{ccc}
1 & 0 & 0 \\
0 & -B_{2} & -B_{3} \\
0 & B_{3} & -B_{2}
\end{array}\right)\left(\begin{array}{l}
x \\
y \\
z
\end{array}\right)
$$


With respect to the coordinates $(\bar{x}, \bar{y}, \bar{z})$, e $(u)$ turns into

$$
\mathbf{e}(u)=\left(\frac{1}{k} \sin (k u), \frac{1}{k} \cos (k u), D\right)
$$

for some constant $D=B_{3} D_{2}-B_{2} D_{3}$ with $D^{2}=1-\frac{1}{k^{2}}$. It is a spherical curve with the curvature $\kappa=\sqrt{1+J^{2}}$ on the unit sphere. Since $J$ is constant, the second equation of (3.13) becomes $Q=J F+a_{0}$ for some constant $a_{0}$. Thus, by the first equation of (3.13) we can find $a_{0} F^{\prime}=0$.

If $a_{0}=0$, then $Q=J F$. In the case, from (3.3) the striction curve $\mathbf{c}(u)$ can be expressed as

$$
\begin{aligned}
\mathbf{c}(u)= & \left(\left(\frac{1}{k}+J D\right) \int F(u) \sin (k u) d u,\left(\frac{1}{k}+J D\right) \int F(u) \cos (k u) d u,\right. \\
& \left.\left(D-\frac{J}{k}\right) \int F(u) d u\right)+\mathbf{d}_{0},
\end{aligned}
$$

where $\mathbf{d}_{0}$ is a constant vector in $\mathbb{E}^{3}$. Thus, up to a rigid motion the evolute offset $\varphi^{*}(u, v)$ of the ruled surface $\varphi(u, v)$ given by (3.16) and (3.15) has the parametrization of the form

$$
\begin{aligned}
\varphi^{*}(u, v)=( & \left(\frac{1}{k}+J D\right) \int F(u) \sin (k u) d u+(R(u)+v) \cos (k u), \\
& \left(\frac{1}{k}+J D\right) \int F(u) \cos (k u) d u-(R(u)+v) \sin (k u), \\
& \left.\left(D-\frac{J}{k}\right) \int F(u) d u\right)+\mathbf{d}_{0} .
\end{aligned}
$$

For specific function $F(u)=u$, the ruled surface $\varphi(u, v)$, generated by (3.15) and (3.16), is showed in Figure 2. Its evolute offset $\varphi^{*}(u, v)$, given by (3.17), is minimal with $J=1, Q=F=u$ and $R=\sin u$ (Figure 3 ).

If $F$ is constant, then $Q$ is also constant. Thus, from (3.3) and (3.15) the striction curve $\mathbf{c}(u)$ becomes

$$
\begin{aligned}
\mathbf{c}(u)= & \left(-\frac{1}{k}\left(\frac{F}{k}+Q D\right) \cos (k u), \frac{1}{k}\left(\frac{F}{k}+Q D\right) \sin (k u),\left(F D-\frac{Q}{k}\right) u\right) \\
& +\mathbf{d}_{0},
\end{aligned}
$$

where $\mathbf{d}_{0}$ is a constant vector in $\mathbb{E}^{3}$. Thus, up to a rigid motion the evolute 


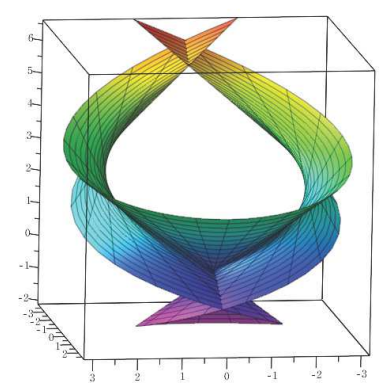

Figure 2.

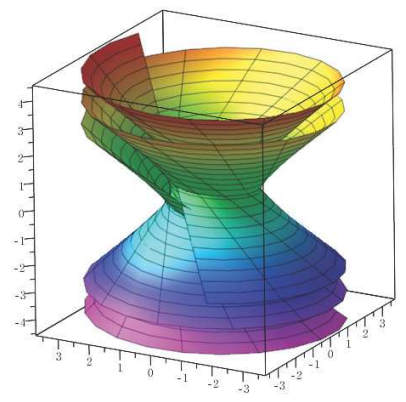

Figure 4 .

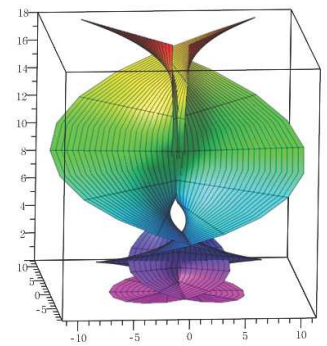

Figure 3 .

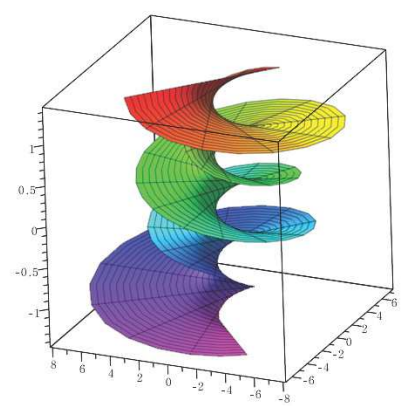

Figure 5.

offset $\varphi^{*}(u, v)$ of the ruled surface $\varphi(u, v)$ has the parametrization of the form

$$
\begin{aligned}
\varphi^{*}(u, v)= & \left(\left(-\frac{F}{k^{2}}-\frac{Q D}{k}+R(u)+v\right) \cos (k u),\right. \\
& \left.\left(\frac{F}{k^{2}}+\frac{Q D}{k}-R(u)-v\right) \sin (k u),\left(F D-\frac{Q}{k}\right) u\right) .
\end{aligned}
$$

In Figure 4, we present the ruled surface $\varphi(u, v)$, generated by $(3.15)$ and (3.18). Figure 5 is showed its evolute offset given by (3.19) with $J=1, Q=$ $1, F=\sqrt{2}$ and $R=2 \cos u$.

Consequently, we have

Theorem 5. If an evolute offset $\varphi^{*}(u, v)$ of a non-developable ruled surface 
$\varphi(u, v)$ in $\mathbb{E}^{3}$ has a constant mean curvature, then $\varphi^{*}(u, v)$ has a zero mean curvature.

Furthermore, an evolute offset $\varphi^{*}(u, v)$ of $\varphi(u, v)$ has a zero mean curvature if and only if the structure functions satisfy either $Q=J F$ and $J$ is constant or $J, F, Q$ are constant.

Theorem 6. An evolute offset $\varphi^{*}(u, v)$ parameterized by (3.17) is only minimal surface which the structure functions $J, F$ and $Q$ of a non-developable ruled surface $\varphi(u, v)$ are $Q=J F$ and $J=$ constant.

Theorem 7. An evolute offset $\varphi^{*}(u, v)$ parameterized by (3.19) is only minimal surface which the structure functions $J, F$ and $Q$ of a non-developable ruled surface $\varphi(u, v)$ are constant.

If a non-developable ruled surface $\varphi(u, v)$ is minimal, then $J=F=0$ and $Q^{\prime}=0$. Thus, the following theorem holds:

Theorem 8. An evolute offset of a minimal non-developable ruled surface is minimal.

\section{Linear Weingarten Offsets of Ruled Surfaces}

In this section, we study a linear Weingarten offset of a non-developable ruled surface in Euclidean 3-space $\mathbb{E}^{3}$.

Let $\varphi^{*}(u, v)$ be an evolute offset of $\varphi(u, v)$ satisfying

$$
a K^{*}+b H^{*}=c
$$

where $a, b, c$ are constant with $(a, b, c) \neq(0,0,0)$. Then from (3.6) and (3.7) we have

$$
b^{2} D^{* 2} H_{1}^{* 2}-\left(2 a(Q-F J)^{2}+2 c D^{* 4}\right)^{2}=0 .
$$

On the other hand, (4.2) is a polynomial in $v$ with functions of $u$ as coefficients. Thus, all the coefficients must be zero. The coefficient of the highest degree $v^{8}$ of the left hand side of (4.2) comes from

$$
4 c^{2} D^{* 8}
$$

and is given by

$$
4 c^{2}\left(1+J^{2}\right)^{4} .
$$


From this, $c=0$, it follows that (4.2) can be rewritten as

$$
b^{2} D^{* 2} H_{1}^{* 2}-4 a^{2}(Q-F J)^{4}=0 .
$$

Also, the coefficient of the term $v^{6}$ in $(4.3)$ must be zero, that is

$$
b^{2}\left(1+J^{2}\right) J^{\prime 2}=0
$$

which yields $b J^{\prime}=0$.

If $J$ is constant, the coefficient of $v^{4}$ in $(4.3)$ is $b^{2}\left(1+J^{2}\right)\left(F^{\prime} J-Q^{\prime}\right)$. So, we get $Q^{\prime}=F^{\prime} J$, which implies from the coefficients of $v^{2}, v^{1}$ and $v^{0}$ we infer that $Q=J F$. According to Theorem 3.7, $\varphi^{*}(u, v)$ is minimal.

If $b=0$, from (4.3) the structure functions satisfy $Q=J F$ because of $a \neq 0$. Thus, the surface $\varphi^{*}(u, v)$ is flat.

Theorem 9. Let $\varphi^{*}(u, v)$ be an evolute offset of a non-developable ruled surface $\varphi(u, v)$ in Euclidean 3-space $\mathbb{E}^{3}$. If $\varphi^{*}(u, v)$ is a linear Weingarten surface, then $\varphi^{*}(u, v)$ is either flat or parameterized as (3.17).

Acknowledgements. The author was supported by Basic Science Research Program through the National Research Foundation of Korea(NRF) funded by the Ministry of Education (2015R1D1A1

A01060046)

\section{References}

[1] A. C. Asperti, B. C. Valério, Ruled Weingarten hypersurfaces in $\mathbb{S}^{n+1}$. Adv. Geom. 8 (2008), 1-10.

[2] A. Barros, J. Silva, P. Sousa, Rotational linear Weingarten surfaces into the Euclidean sphere. Israel J. Math. 192 (2012), 819-830.

[3] E. Beltrami, Risoluzione di un Problema Relativo alla Teoria delle Superficie Gobbe. Ann. Mat. Pura Appl. 7 (1865/1866), 139-150.

[4] M. Dajczer, K. Tenenblat, Rigidity for complete Weingarten hypersurfaces. Trans. Amer. Math. Soc. 312 (1989), 129-140.

[5] G. Darboux, Leçons sur la Théorie Général des Surfaces et les Applications Géométriques du Calcul Infinitésimal. Vol III, Gauthier-Villars 1894.

[6] U. Dini, Sulle Superficie Gobbe nelle quali uno dei due Raggi di Curvatura Principale é una Funzione Dell'altro. Ann. Mat. Pura Appl. 7 (1865/1866), 205-210.

[7] J. A. Gaĺvez, A. Martińez, F. Milań, Linear Weingarten surfaces in $\mathbb{R}^{3}$. Monatsh. Math. 138 (2003), 133-144.

[8] E. Kasap, S. Yuce, N. Kuruoglu, The involute-evolute offsets of ruled surfaces. Iranian Journal of Science \& Technology, Transaction A 33 (2009), 195-201. 
[9] E. Kruppa, Analytische und konstruktive Differentialgeometrie. Springer, Berlin 1957.

[10] W. Kühnel, Differentialgeometrie. Vieweg, Berlin 2008.

[11] W. Kühnel, Ruled W-surfaces. Arch. Math. 62 (1994), 475-480.

[12] S. Lie, Über Flächen deren Krümmungsradien durch eine Relation verknüpft sind Arch. Math. 4 (1880), 507-512.

[13] H. Liu, Y. Yu, S. D. Jung, Invariants of non-developable ruled surfaces in Euclidean 3-space. Beitr. Alegebra Geom. 55 (2014), 189-199.

[14] R. Lopez, Rotational linear Weingarten surfaces of hyperbolic type. Israel J. Math. 167 (2008), 283-301.

[15] A. Lymberopoulos, Hipersuperficies regradas e de Weingarten no espaco hiperbólico. Ph.D. Thesis, IME-USP, São Paulo 2009.

[16] Y. Tuncer, D. W. Yoon, M. K. Karacan, Weingarten and linear Weingarten type tubular surfaces in $\mathbb{E}^{3}$ Math. Problems in Engineering Article ID 191849 (2011).

[17] C. V. Valério, Hipersuperficies regadas e de Weingarten em formas espacias. Ph.D. Thesis, IME-USP, São Paulo (2004).

[18] J. Weingarten, Über eine Klasse auf einander abwickelbarer Flächen. J. Reine Angew. Math. 59 (1861), 382-393.

[19] D. W. Yoon, Y. Tuncer, M. K. Karacan, Non-degenerate quadric surfaces of Weingarten type. Annales Polonici Math. 107 (2013), 59-69. 
